\title{
Clinical screening assay for $E G F R$ exon 19 mutations using PNA-clamp smart amplification process version 2 in lung adenocarcinoma
}

\author{
TAKUYA ARAKI ${ }^{1,4}$, KIMIHIRO SHIMIZU ${ }^{2}$, TOMONORI NAKAMURA ${ }^{1,4}$, MASARU BABA $^{5,6}$, YUKI KAWAI ${ }^{5,6}$, \\ KATSUNORI NAKAMURA $^{1}$, YASUMASA MITANI ${ }^{5,6}$, KYOKO OBAYASHI $^{4}$, TOHRU AOMORI $^{4}$, \\ YUKIYOSHI FUJITA ${ }^{4}$, YOHEI MIYAMAE ${ }^{2}$, SEIICHI KAKEGAWA ${ }^{2}$, KYOICHI KAIRA $^{3}$, ALEXANDER LEZHAVA ${ }^{6}$, \\ YOSHIHIDE HAYASHIZAKI ${ }^{6}$, IZUMI TAKEYOSHI ${ }^{2}$ and KOUJIROU YAMAMOTO ${ }^{1,4}$
}

\begin{abstract}
Departments of ${ }^{1}$ Clinical Pharmacology, ${ }^{2}$ Thoracic and Visceral Organ Surgery, and ${ }^{3}$ Medicine and Molecular Science, Gunma University Graduate School of Medicine, Maebashi 371-8511; ${ }^{4}$ Department of Pharmacy, Gunma University Hospital, Maebashi 371-8511; ${ }^{5}$ K.K. DNAFORM, Tsurumi-ku, Yokohama, Kanagawa 230-0046;

${ }^{6}$ Omics Sciences Center (OSC), RIKEN Yokohama Institute, Tsurumi-ku, Yokohama 230-0045, Japan
\end{abstract}

Received March 14, 2011; Accepted May 6, 2011

DOI: $10.3892 / o r .2011 .1391$

\begin{abstract}
The presence of EGFR mutations is correlated with a positive therapeutic response to tyrosine kinase inhibitors; therefore, the accurate detection of EGFR mutations is crucial when deciding appropriate therapeutic strategies. Recently, the rapid and sensitive assay smart amplification process version 2 (SmartAmp2) was developed. However, this method can only detect one type of mutation in EGFR exon 19; therefore, we applied the PNA technology to the SmartAmp2 assay to develop PNA-clamp SmartAmp2 for the detection of many types of deletions in EGFR exon 19, in a single reaction. This new assay was evaluated using 172 clinical samples. Thirty-nine $(22.7 \%)$ samples were found to have deletions by PNA-clamp SmartAmp2; whereas 30 (17.4\%) and 38 (22.1\%) tumors were found to have deletions by direct sequencing and PNA-enriched sequencing, respectively. Three cases, in which we detected mutations with PNA-clamp SmartAmp2, but not with direct sequencing, were treated with gefitinib, and all cases showed a partial therapeutic response. Using clinical samples, we demonstrated that PNA-clamp SmartAmp2 can detect various types of mutations in EGFR exon 19 in a relatively short time and with high sensitivity. This method detected small amounts of mutant DNA and identified patients for whom clinical information was previously unavailable from other tests. This test may contribute to the administration of efficient therapeutic strategies.
\end{abstract}

Correspondence to: Dr Takuya Araki, Department of Clinical Pharmacology, Gunma University Graduate School of Medicine, 3-39-22 Showa-machi, Maebashi 371-8511, Japan

E-mail: tkyaraki@gunma-u.ac.jp

Key words: EGFR mutation, non-small cell lung cancer,PNA-clamp SmartAmp2

\section{Introduction}

The epidermal growth factor receptor (EGFR) tyrosine kinase inhibitor (TKI) gefitinib has emerged as an effective therapeutic agent for some patients with advanced non-small cell lung cancer (NSCLC) (1). Recently, a significant association between somatic mutations in the EGFR gene and dramatic positive clinical responses to TKIs, such as gefitinib and elrotinib, has been reported in NSCLC patients $(2,3)$. Approximately $85-90 \%$ of these mutations occur in exons 19 and 21 near the ATP cleft of the tyrosine kinase domain (2-6). Therefore, information concerning somatic mutation of the EGFR gene in lung cancer cells is very useful for physicians to design optimal therapeutic strategies for NSCLC patients.

Recently, several highly sensitive methods, such as mutantenriched assays, the PCR-invader method, cycleave-PCR assay, Scorpion-Amplified refractory mutation system assay, TaqMan PCR assay, denaturing high-performance liquid chromatography method, high resolution melting assay, highresolution chipCE assay, immunohistochemistry (IHC) assay, and the peptide nucleic acid-locked nucleic acid (PNA-LNA) PCR clamp method, have been reported for the detection of EGFR mutations (7-25). Some of these techniques have quite high sensitivity, but they are still not ideal for routine clinical use in general hospitals or outpatient clinics due to various reasons such as long turn-around times and complexity.

In 2007, the rapid, simple and sensitive mutation detection assays, smart amplification process version 2 (SmartAmp2) and PNA-clamp SmartAmp2 for SNP detection, were developed $(26,27)$. The SmartAmp2 method is a unique genotyping technology that can detect a mutation within 30 min under isothermal conditions and in a single step. This method was applied to the detection of one specific deletion of EGFR exon 19, del 2235-2249 (del E746-A750 DEL) (28). However, since many types of mutations in exon 19 are associated with the response to gefitinib, the SmartAmp2 

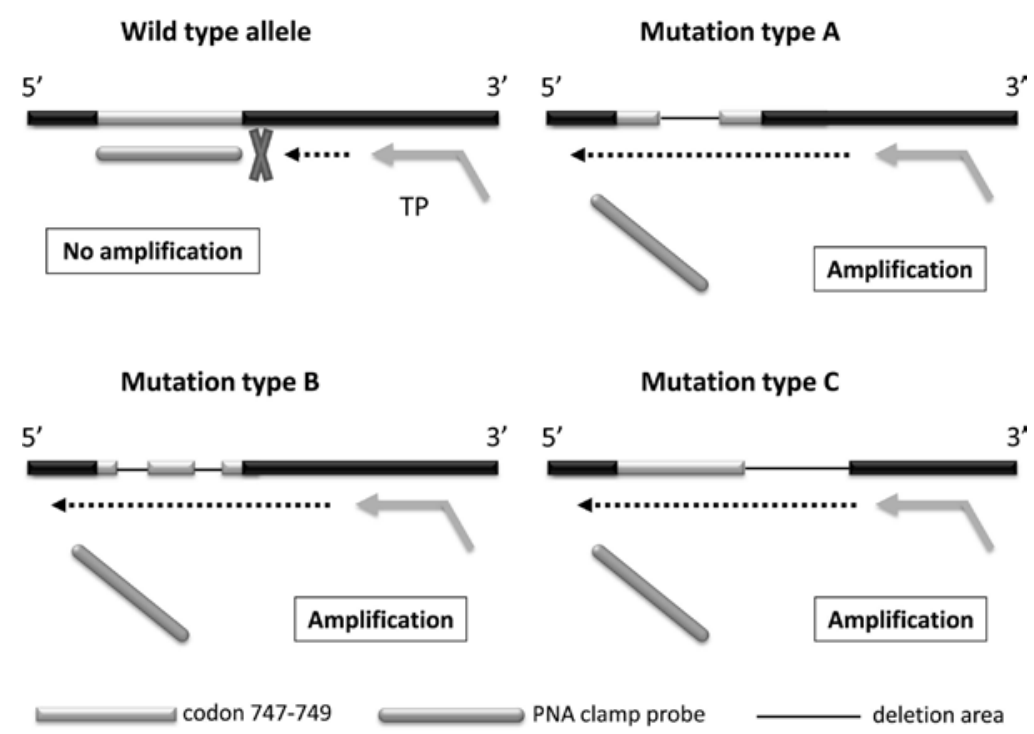

Figure 1. Principle of PNA-clamp SmartAmp2 for detection of EGFR exon 19 deletions. The PNA-clamp competitive probe is designed for the wild-type allele sequence at codon 747-749. The greater stability of the PNA probe during hybridization inhibits wild-type allele amplification. Amplification of the mutant allele is not inhibited by PNA.

assay still has a low performance and is less desirable for clinical screening. Therefore, we adopted PNA technology, which clamps to the commonly deleted sequences containing codons 747-749 (6), to SmartAmp2 and developed PNA-clamp SmartAmp2 for detection of a range of different deletions in EGFR exon 19 in one reaction, not only for the detection of SNPs. Furthermore, we compared this method with PCR-based direct sequencing and PNA-enriched sequencing using clinical samples from 172 patients with lung adenocarcinoma and demonstrated that the PNA-clamp SmartAmp2 assay has high sensitivity and is a very useful and reliable tool for the clinical screening of EGFR exon 19 deletions.

\section{Materials and methods}

Clinical samples, cell lines, and DNA extraction. Tumor samples containing EGFR mutations obtained from 172 consecutive patients with lung adenocarcinoma who were surgically treated at Gunma University Hospital (Gunma, Japan) between September 2002 and December 2008 were enrolled in this study. Thirty non-malignant specimens from peripheral lung tissue surrounding the tumors were used to examine the specificity of the assays. This study was conducted in accordance with the Declaration of Helsinki and its amendments, and was approved by the Institutional Review Board for Clinical Trials at Gunma University Hospital and the Ethics Committee for Human Genome Analysis at Gunma University. Written consent was obtained from all participants after they had been informed of the experimental procedure and the purpose of the study. All tumor tissues were diagnosed as lung cancer by hematoxylin and eosin staining. After surgical removal, all tumor samples were immediately frozen and stored at $-80^{\circ} \mathrm{C}$.

To assist in the comparison of mutation detection methods, we used DNA harboring EGFR mutations derived from the PC-9 lung cancer cell line, which contains the del 2235-2249 EGFR exon 19 mutation (del E746-A750). In addition, DNA containing wild-type EGFR was derived from the A549 human alveolar epithelial cell line. The gene sequences of EGFR exon 19 obtained from each cell line were confirmed by PCR-based direct sequencing.

DNA samples were extracted from tumor tissues and cell lines using the QIAamp DNA Mini Kit (Qiagen Inc., Valencia, CA, USA) according to the manufacturer's instructions and were serially diluted to a concentration of $20 \mathrm{ng} / \mu \mathrm{l}$. For the evaluation of sensitivity, DNA from the PC-9 cell line was diluted with DNA from the A549 cell line to give mutationwild-type ratios of $50,10,5,1,0.5$ and $0.1 \%$. The DNA templates extracted from the tumor samples and cell lines were stored at $-20^{\circ} \mathrm{C}$.

PNA-clamp SmartAmp2 and established SmartAmp2. We performed the established SmartAmp2 using an EGFR detection kit (K.K. DNAFORM; Kanagawa, Japan) to detect mutations in EGFR exon 19, according to the manufacturer's instructions. The principles of the SmartAmp2 have been previously described (26). We designed the PNA-clamp SmartAmp2 so it would amplify almost all types of deletions that occur in the hot spot of the mutation in EGFR exon 19. The primers were modified, and the 18-bp PNA clamp primer was designed to fully match the wild-type sequence so that it spanned the commonly deleted region (codon 747-749). Consequently, hybridization of the wild-type PNA clamp primer inhibited chain elongation from the turn-back primer, resulting in suppressed amplification of the wild-type allele (Fig. 1). The master mix and primer mix and PNA-clamp primers were prepared according to previous reports $(26,27)$ by the company K.K. DNAFORM. The reaction was performed in a total volume of $25 \mu \mathrm{l}$ containing $1 \mathrm{X}$ master mix, $2 \mu \mathrm{l}$ primer mix, $1 \mu \mathrm{l}$ Aac DNA Polymerase, and $40 \mathrm{ng}$ genomic DNA. The PNA-clamp SmartAmp2 and established SmartAmp2 assay reactions were assembled on ice and incubated at $60^{\circ} \mathrm{C}$ for $60 \mathrm{~min}$. The Mx3000P system (Stratagene, La Jolla, CA, USA) was used to maintain isothermal 
A

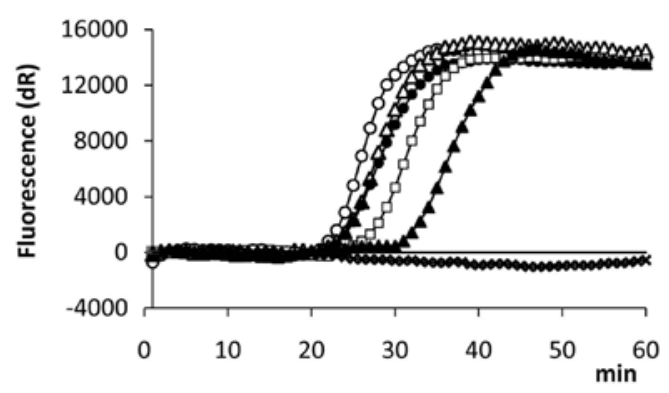

O : $50 \%$ mutation

$\Delta: 10 \%$ mutation

: $5 \%$ mutation

$\square: 1 \%$ mutation

$\mathbf{\Delta}: 0.5 \%$ mutation

$\mathbf{X}$ : wild type

B

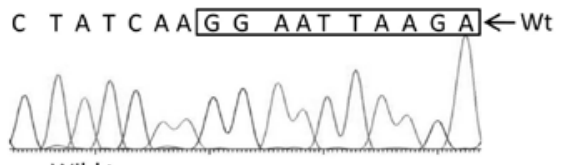

Wild type

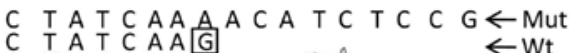

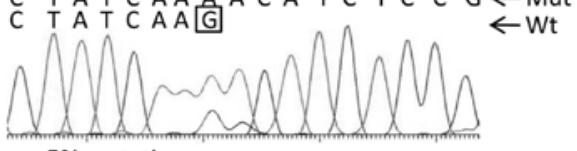

$5 \%$ mutation

C TATCAAA A C A T C TC C G

T A T C A A C ATC TC C G

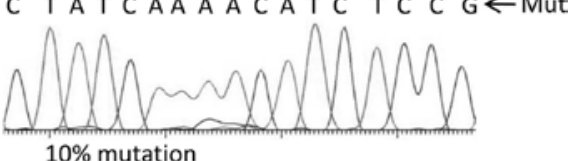

$10 \%$ mutation

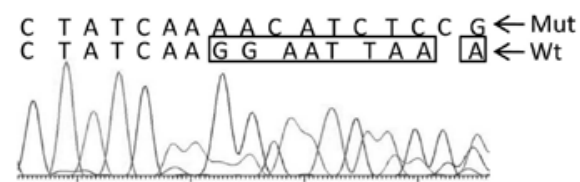

$1 \%$ mutation

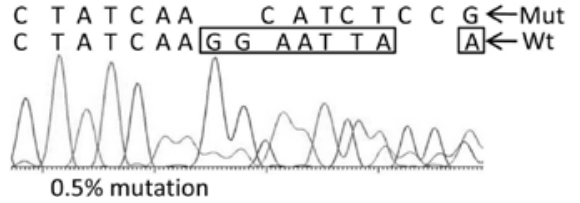

Figure 2. Evaluation of the sensitivity of each detection method for EGFR exon 19 deletions. (A) Sensitivity of PNA-clamp SmartAmp2. (B) Sensitivity of PNA-enriched sequencing.

conditions and monitor the transition of fluorescence intensity of intercalating SYBR-Green I (Invitrogen, Tokyo, Japan) during the reactions. We evaluated the results of the PNA-clamp SmartAmp2 and established SmartAmp2 assays according to the criterion of amplification versus nonamplification within $60 \mathrm{~min}$. Each DNA sample was analyzed in triplicate.

PNA-enriched sequencing. We identified EGFR exon 19 deletions by PNA-enriched sequencing (29). PNA-enriched PCR was performed in a total volume of $25 \mu \mathrm{l}$ containing 1X PCR Gold Buffer, $1.5 \mathrm{mmol} / 1 \mathrm{MgCl}_{2}, 200 \mu \mathrm{mol} / 1 \mathrm{dNTPs}$, $500 \mathrm{nmol} / 1$ each primer, $1 \mu \mathrm{mol} / 1$ PNA clamp primer, $1 \mathrm{U}$ Taq DNA Gold polymerase (Applied Biosystems, CA, USA), and 20 ng genomic DNA. The PNA clamp primer was designed to be exactly homologous to the wild-type allele at codons 746-751. Thermal cycling conditions included a pre-incubation step at $94^{\circ} \mathrm{C}$ for $5 \mathrm{~min}$, followed by 40 cycles at $94^{\circ} \mathrm{C}$ for $15 \mathrm{sec}, 60^{\circ} \mathrm{C}$ for $30 \mathrm{sec}, 72^{\circ} \mathrm{C}$ for $1 \mathrm{~min}$, and a final extension at $72^{\circ} \mathrm{C}$ for $5 \mathrm{~min}$. The PCR products were then purified using the QIAquick PCR Purification Kit (Qiagen). DNA sequencing was performed with the ABI PRISM 3100 DNA Analyzer (Applied Biosystems) using the ABI PRISM BigDye Terminator version 3.1 (Applied Biosystems).

PCR-based direct sequencing. We performed PCR-based direct sequencing. The PCR conditions were the same as for the PNA-enriched PCR, but without the PNA clamp primer.
The PCR products were then purified and sequenced under the aforementioned conditions.

PNA-clamp SmartAmp2-based sequencing. We performed PNA-clamp SmartAmp2-based sequencing on 2 samples in which mutations were detected by the PNA-clamp SmartAmp2 assay, but not by the other PCR-based methods. The PNA-clamp SmartAmp2 products were diluted 1000-fold with distilled water, and $1 \mu 1$ of the diluted product was processed for PCR. The PCR reactions were performed in a total volume of $20 \mu \mathrm{l}$ containing $1 \mathrm{X}$ PCR Gold Buffer, $1.3 \mathrm{mmol} / 1 \mathrm{MgCl}_{2}, 200 \mu \mathrm{mol} / 1 \mathrm{dNTPs}, 500 \mathrm{nmol} / 1$ of each primer (ex19-SF and ex19-SR), 1 U Taq DNA Gold polymerase, and $1 \mu \mathrm{l}$ diluted DNA. Thermal cycling conditions included a pre-incubation step at $94^{\circ} \mathrm{C}$ for $5 \mathrm{~min}$, followed by 35 cycles at $94^{\circ} \mathrm{C}$ for $30 \mathrm{sec}, 52^{\circ} \mathrm{C}$ for $30 \mathrm{sec}$, and $72^{\circ} \mathrm{C}$ for $30 \mathrm{sec}$, and a final extension at $72^{\circ} \mathrm{C}$ for $7 \mathrm{~min}$. The PCR products were purified and sequenced under the aforementioned conditions.

\section{Results}

Detection sensitivity. To evaluate the sensitivity of PNA-clamp SmartAmp2 for the detection of EGFR exon 19 deletions, we used serially diluted DNA obtained from lung cancer cell lines containing EGFR mutant and wild-type genes. The results of PNA-enriched sequencing and the PNA-clamp SmartAmp2 method for the detection of EGFR exon 19 
Table I. Comparison of mutation detection methods in the clinical samples.

\begin{tabular}{|c|c|c|c|c|}
\hline Case & PNA-clamp SmartAmp2 & Established SmartAmp2 & Direct sequencing & PNA-enriched sequencing \\
\hline $19-1$ & Mutant-type & Wild-type & L747-T751 del & L747-T751 del \\
\hline $19-2$ & Mutant-type & E746-A750 del (type 1) & E746-A750 del (type 1) & E746-A750 del (type 1) \\
\hline $19-3$ & Mutant-type & Wild-type & E746-A750 del (type 2) & E746-A750 del (type 2) \\
\hline $19-4$ & Mutant-type & Wild-type & Wild-type & L747-T751del \\
\hline $19-5$ & Mutant-type & E746-A750 del (type 1) & E746-A750 del (type 1) & E746-A750 del (type 1) \\
\hline $19-6$ & Mutant-type & E746-A750 del (type 1) & E746-A750 del (type 1) & E746-A750 del (type 1) \\
\hline $19-7$ & Mutant-type & E746-A750 del (type 1) & Wild-type & E746-A750 del (type 1) \\
\hline $19-8$ & Mutant-type & Wild-type & L747-E749 del, A750P & L747-E749 del, A750P \\
\hline $19-9$ & Wild-type & Wild-type & 26 bp deletion $+\mathrm{AT}$ ins & $26 \mathrm{bp}$ deletion $+\mathrm{AT}$ ins \\
\hline $19-10$ & Mutant-type & Wild-type & E746-A750 del (type 2) & E746-A750 del (type 2) \\
\hline $19-11$ & Mutant-type & Wild-type & Wild-type & E746-A750 del (type 2) \\
\hline $19-12$ & Mutant-type & Wild-type & L747-E749del, A750P & L747-E749del, A750P \\
\hline $19-13$ & Mutant-type & Wild-type & E746-A750 del (type 2) & E746-A750 del (type 2) \\
\hline $19-14$ & Mutant-type & Wild-type & E746-A750 del (type 2) & E746-A750 del (type 2) \\
\hline $19-15$ & Mutant-type & Wild-type & Wild-type & E746-E749, S752-T753 \\
\hline $19-16$ & Mutant-type & Wild-type & L747-E749 del, A750P & L747-E749 del, A750P \\
\hline $19-17$ & Mutant-type & Wild-type & Wild-type & E746-A750 del (type 2) \\
\hline $19-18$ & Mutant-type & E746-A750 del (type 1) & E746-A750 del (type 1) & E746-A750 del (type 1) \\
\hline $19-19$ & Mutant-type & E746-A750 del (type 1) & E746-A750 del (type 1) & E746-A750 del (type 1) \\
\hline $19-20$ & Mutant-type & Wild-type & L747-S752 del & L747-S752 del \\
\hline $19-21$ & Mutant-type & Wild-type & E746-A750 del (type 2) & E746-A750 del (type 2) \\
\hline $19-22$ & Mutant-type & Wild-type & E746-A750 del (type 2) & E746-A750 del (type 2) \\
\hline $19-23$ & Mutant-type & Wild-type & L747-E749 del, A750P & L747-E749 del, A750P \\
\hline $19-24$ & Mutant-type & Wild-type & E746-E749, T751-P753 del & E746-E749, T751-P753 del \\
\hline $19-25$ & Mutant-type & Wild-type & Wild-type & E746-A750 del (type 2) \\
\hline $19-26$ & Mutant-type & Wild-type & E746-A750 del (type 2) & E746-A750 del (type 2) \\
\hline $19-27$ & Mutant-type & Wild-type & L74-E749 del, A750P & L74-E749 del, A750P \\
\hline $19-28$ & Mutant-type & Wild-type & L747-T751 del, K754N & L747-T751 del, K754N \\
\hline $19-29$ & Mutant-type & E746-A750 del (type 1) & E746-A750 del (type 1) & E746-A750 del (type 1) \\
\hline $19-30$ & Mutant-type & Wild-type & E746-A750 del (type 2) & E746-A750 del (type 2) \\
\hline $19-31$ & Mutant-type & E746-A750 del (type 1) & E746-A750 del (type 1) & E746-A750 del (type 1) \\
\hline $19-32$ & Mutant-type & E746-A750 del (type 1) & E746-A750 del (type 1) & E746-A750 del (type 1) \\
\hline $19-33$ & Mutant-type & Wild-type & Wild-type & Wild-type \\
\hline $19-34$ & Mutant-type & Wild-type & Wild-type & L747-S752 del \\
\hline $19-35$ & Mutant-type & E746-A750 del (type 1) & E746-A750 del (type 1) & E746-A750 del (type 1) \\
\hline $19-36$ & Mutant-type & Wild-type & Wild-type & Wild-type \\
\hline $19-37$ & Mutant-type & Wild-type & E746-A750 del (type 2) & E746-A750 del (type 2) \\
\hline $19-38$ & Mutant-type & Wild-type & Wild-type & L747-S752 del \\
\hline $19-39$ & Mutant-type & E746-A750 del (type 1) & E746-A750 del (type 1) & E746-A750 del (type 1) \\
\hline $19-40$ & Mutant-type & E746-A750 del (type 1) & E746-A750 del (type 1) & E746-A750 del (type 1) \\
\hline
\end{tabular}

Type 1, del 2235-2249; type 2, del 2236-2250.

deletions diluted with wild-type DNA are shown in Fig. 2. It was difficult to differentiate these samples containing 0.5 or $1 \%$ mutant DNA from the background noise by PNA-enriched sequencing (Fig. 2B), whereas we clearly detected a sample containing $0.5 \%$ mutant DNA by PNA-clamp SmartAmp2 (Fig. 2A). The detection limits of PNA-enriched sequencing and PCR-based direct sequencing were 1 and $10 \%$ mutant DNA, respectively.
Mutation detection in clinical samples. We screened 172 lung adenocarcinoma samples obtained from Asian patients for mutations in EGFR exon 19. We compared the detection abilities of PCR-based direct sequencing, PNA-enriched sequencing, established SmartAmp2 and PNA-clamp SmartAmp2.

Among the 172 tumor samples, 39 (22.7\%) samples were found to have EGFR exon 19 deletions by PNA-clamp 
A
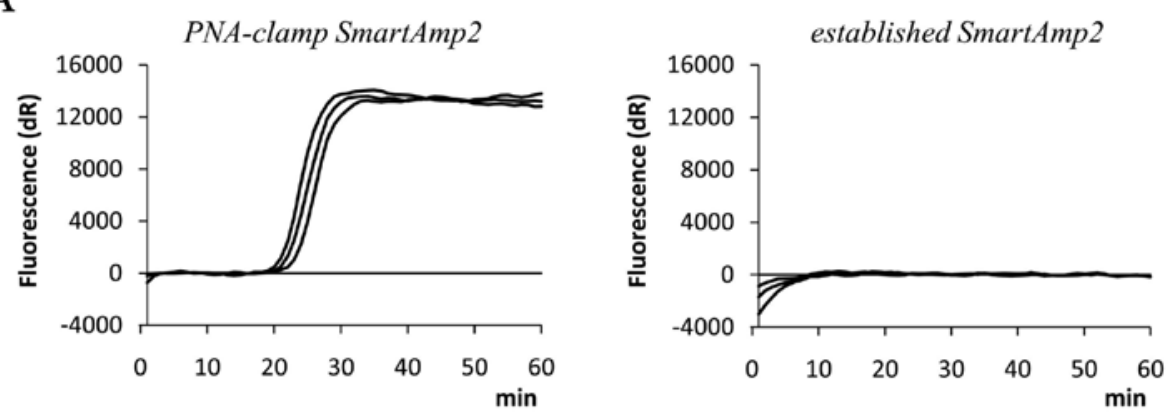

PCR-based direct sequencing

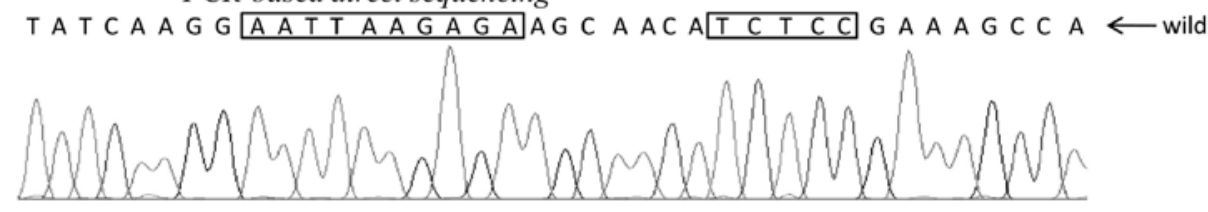

PNA-enriched sequencing

TATCAAG G AAT TAAGAGAAGCAACATCTCCGAAAGCCA

TATCAAG G A G A A A A A A G C A ACA AGGG AATC C T C G

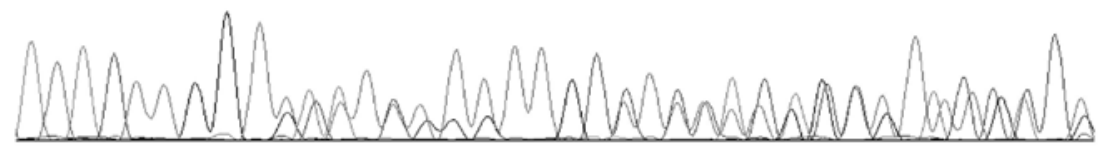

B

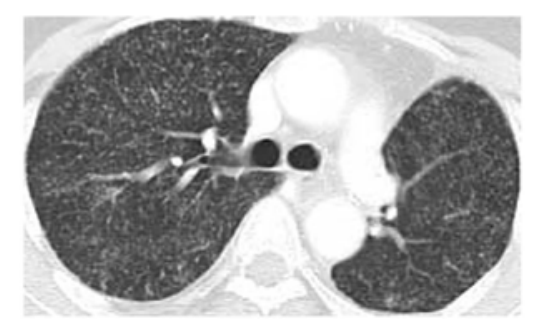

C

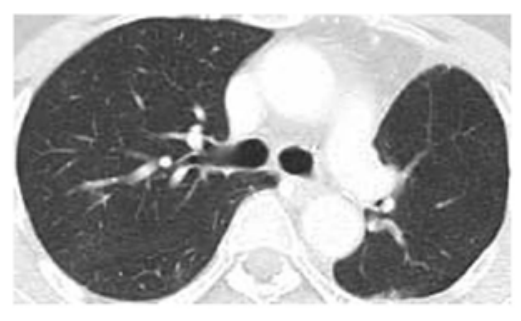

Figure 3. Partial response to gefitinib treatment in patient with EGFR exon 19 deletions (case 19-15). (A) Results using different methods of detection for mutations in EGFR exon 19. The deletion was detected by PNA-clamp SmartAmp2 and PNA-enriched sequencing but not by the established SmartAmp2 assay or PCR-based direct sequencing. (B) Pre-treatment CT scan shows multiple micronodules in the bilateral lung fields. (C) After 3 months of gefitinib administration. Multiple micronodules in the bilateral lung fields were diminished.

SmartAmp2, whereas $30(17.4 \%), 38(22.1 \%)$, and $12(7.0 \%)$ tumors were found to have EGFR exon 19 deletions by PCR-based direct sequencing, PNA-enriched sequencing, and the established SmartAmp2 method, respectively. The 39 (22.7\%) tumors with EGFR exon 19 deletions detected by PNA-clamp SmartAmp2 included 2 tumors that were undetected by the other methods. Only 1 sample was found to have an EGFR exon 19 deletion by PNA-enriched sequencing but not by PNA-clamp SmartAmp2 (case 19-9). A summary of the $40 E G F R$ exon 19 deletions detected in this study is presented in Table I. The deletions in the 39 tumors that were detected by PNA-clamp SmartAmp2 were verified by PNA-clamp SmartAmp2-based sequencing, and all tumors were confirmed to be harboring EGFR exon 19 deletions.

We also examined 30 peripheral lung tissue specimens as non-malignant samples and found no exon 19 mutations in any of the samples using the PNA-clamp SmartAmp2 and established SmartAmp2 assays.
Screening capacity. In this study, we identified 9 types of deletions (40 tumors) by PNA-enriched sequencing or PNA-clamp SmartAmp2-based sequencing and we detected 8 types (39 tumors) by the PNA-clamp SmartAmp2 assay, whereas only 1 type (12 tumors) could be detected by the established SmartAmp2 assay (Table I). The PNA-clamp SmartAmp2 failed to detect del 2252-2277 deletion because this deletion area contained the primer binding site.

In addition to detecting these mutations, PNA-clamp SmartAmp2 provided reproducible amplification curves when each sample was examined in triplicate. Furthermore, performing the PNA-clamp SmartAmp2 assay required only 60 min, whereas PNA-enriched sequencing required more than $9 \mathrm{~h}$ to detect $E G F R$ exon 19 mutations.

Low amounts of mutant EGFR DNA and the gefitinib response. Of the 10 cases in which we detected EGFR exon 19 deletions by PNA-clamp SmartAmp2, but not by PCR-based 
direct sequencing, 3 cases (cases 19-7, 19-15 and 19-25) were treated with gefitinib according to the patients' wishes. All cases showed a partial response to gefitinib. Case 19-7 carried a major deletion (del 2235-2249) and was detected by PNA-clamp SmartAmp2 and the established SmartAmp2 method. However, cases 19-15 and 19-25 carried a minor deletion that was detected by PNA-clamp SmartAmp2, but not by the established SmartAmp2. The results of mutation detection by each method and the results of a computed tomography scan taken before and after gefitinib treatment are shown in Fig. 3. Seven additional patients were treated with other chemotherapy regimens according to their wishes.

\section{Discussion}

The rapid, simple and sensitive SNP detection assay, SmartAmp2, has been recently developed (26). Although the SmartAmp2 assay has been applied to the detection of EGFR exon 19 deletions, it can detect only one specific deletion of EGFR exon 19, del 2235-2249 (28). Since many types of exon 19 mutations are associated with the therapeutic response to gefitinib, the established SmartAmp2 assay has a low performance in clinical screening.

In this study, we modified the primers and adopted PNA as a clamp primer for SmartAmp2 and developed the PNA-clamp SmartAmp2 assay to detect many types of EGFR exon 19 deletions, not only SNPs, in one tube and one reaction. We also assessed the usefulness of this technique for routine clinical diagnosis. We applied this technique to the detection of $E G F R$ exon 19 deletions in tumor samples from 172 patients with lung adenocarcinoma and compared it to other conventional methods and the established SmartAmp2 assay.

In evaluating the 172 clinical samples, PNA-clamp SmartAmp2 had a similarly high-sensitivity (39/172) as the PNA-enriched sequencing (37/172). We evaluated the slides from tumors with the mutant EGFR gene that were detected by PNA-clamp SmartAmp2 but not by PCR-based methods and observed that they had a lower percentage of tumor cells or more marked fibrosis than tumors that were detected by PNA-clamp SmartAmp2 and PCR-sequencing. Thus, the higher sensitivity of the PNA-clamp SmartAmp2 assay may make it more suitable for detecting the presence of small amounts of DNA containing EGFR mutations in fibrotic samples.

However, there was one mutant sample that was not detected by PNA-clamp SmartAmp2, but was detected by PCR-based direct sequencing and PNA-enriched sequencing. This mutation was a fairly minor type, and this false-negative appeared to be the result of a limitation of PNA technology; the PNA designed for PNA-clamp SmartAmp2 did not include this exceedingly rare deletion area. If this mutation is also associated with the response to TKIs and is clinically significant, the primer and PNA clamp primer should be redesigned and another new assay kit should be developed to detect this rare deletion. The other 96 patients who were identified with the wild-type EGFR exon 19 allele by PNA-clamp SmartAmp2 were also shown to have the wild-type allele by the other methods. In the future, as no strong evidence exists concerning the appropriate sensitivity of genetic testing methods to identify responders, the appropriate sensitivity with which to identify real responders must be clarified so more suitable diagnostic methods can be developed.

We previously reported that the SmartAmp2 assay effectively detects mutations in DNA extracted from formalinfixed paraffin-embedded (FFPE) tissue because of this method's ability to amplify quite short sequences (30). We believe that the advantages of PNA-clamp SmartAmp2, its rapid detection time and ability to detect mutations in FFPE tissue, will enable this method to be particularly useful in the treatment of outpatients. If the conventional methods are utilized, patients who wish to receive EGFR-TKI treatment must come to the hospital twice; initially to receive information concerning EGFR-TKI treatment and to undergo genetic testing, and a second time to receive the results of the genetic testing and to start EGFR-TKI treatment. Conversely, if $E G F R$ mutations can be determined within a short time period using PNA-clamp SmartAmp2, which is conceivable as this test can be performed in the hospital, patients with EGFR mutations can immediately receive appropriate treatment without any unnecessary waiting. Thus, the advantages of the PNA-clamp SmartAmp2 assay will improve the efficacy of EGFR-TKI and other treatments and improve the quality of life of patients by minimizing the waiting time until the initiation of the appropriate treatment. Recently, the presence of EGFR mutations in plasma, serum, and pleural effusion has become a focus of interest (31-34). The PNA-clamp SmartAmp2 assay may also contribute to the development of mutant DNA detection methods in these specimens, which contain extremely small amounts of mutant cells.

In conclusion, we adopted PNA, which clamps to the commonly deleted sequence containing codons 747-749 for the SmartAmp2 assay and developed PNA-clamp SmartAmp2 for detection of a wide range of deletions in EGFR exon 19 in one reaction. We detected small amounts of mutant DNA by this method that were not detected by traditional methods and identified patients for whom this assay provided clinical information that was unavailable from the other available tests, thereby contributing to the design of appropriate therapeutic strategies. The sensitivity of this assay highlights the need to clarify the association between the effects of TKIs and low levels of mutant EGFR alleles, and points to important new areas of study. The PNA-clamp SmartAmp2 should be developed to detect mutations in EGFR exons other than exon 19. The advantages and reliability of this method for the clinical diagnosis of EGFR mutations in lung cancer may contribute to the effective and safe use of lung cancer pharmacotherapies.

\section{Acknowledgements}

We thank Ms. Aiko Matsumoto for the secretarial assistance and Ms. Tomoko Senbongi for the technical assistance. This study was supported by a Grant-in-Aid for Scientific Research (KAKENHI 19390359) from the Japan Society for the Promotion of Science.

\section{References}

1. Cohen MH, Williams GA, Sridhara R, et al: United States Food and Drug Administration drug approval summary: Gefitinib (ZD1839; Iressa) tablets. Clin Cancer Res 10: 1212-1218, 2004. 
2. Lynch TJ, Bell DW, Sordella R, et al: Activating mutations in the epidermal growth factor receptor underlying responsiveness of non-small cell lung cancer to gefitinib. N Engl J Med 350: 2129-2139, 2004

3. Paez JG, Jänne PA, Lee JC, et al: EGFR mutations in lung cancer: correlation with clinical response to gefitinib therapy. Science 304: 1497-1500, 2004

4. Huang SF, Liu HP, Li LH, et al: High frequency of epidermal growth factor receptor mutations with complex patterns in non-small cell lung cancers related to gefitinib responsiveness in Taiwan. Clin Cancer Res 10: 8195-8203, 2004.

5. Kosaka T, Yatabe Y, Endoh H, Kuwano H, Takahashi T and Mitsudomi T: Mutations of the epidermal growth factor receptor gene in lung cancer: biological and clinical implications. Cancer Res 64: 8919-8923, 2004.

6. Tokumo M, Toyooka S, Kiura K, et al: The relationship between epidermal growth factor receptor mutations and clinicopathologic features in non-small cell lung cancers. Clin Cancer Res 11: 1167-1173, 2005.

7. Asano H, Toyooka S, Tokumo M, et al: Detection of EGFR gene mutation in lung cancer by mutant enriched polymerase chain reaction assay. Clin Cancer Res 12: 43-48, 2006.

8. Cohen V, Agulnik JS, Jarry J, et al: Evaluation of denaturing high-performance liquid chromatography as a rapid detection method for identification of epidermal growth factor receptor mutations in non-small cell lung cancer. Cancer 107: 2858-2865, 2006.

9. Endo K, Konishi A, Sasaki H, et al: Epidermal growth factor receptor gene mutation in non-small cell lung cancer using highly sensitive and fast TaqMan PCR assay. Lung Cancer 50 : 375-384, 2005

10. Jänne PA, Borras AM, Kuang Y, et al: A rapid and sensitive enzymatic method for epidermal growth factor receptor mutation screening. Clin Cancer Res 12: 751-758, 2006.

11. Kimura H, Kasahara K, Kawaishi M, Kunitoh H, Tamura T, Holloway B and Nishio K: Detection of epidermal growth factor receptor mutations in serum as a predictor of the response to gefitinib in patients with non-small cell lung cancer. Clin Cancer Res 12: 3915-3921, 2006.

12. Kimura H, Fujiwara Y, Sone T, Kunitoh H, Tamura T, Kasahara K and Nishio K: High sensitivity detection of epidermal growth factor receptor mutations in the pleural effusion of non-small cell lung cancer patients. Cancer Sci 97: 642-648, 2006.

13. Lin CH, Yeh KT, Chang YS, Hsu NC and Chang JG: Rapid detection of epidermal growth factor receptor mutations with multiplex PCR and primer extension in lung cancer. J Biomed Sci 12: 37-42, 2010

14. Marchetti A, Martella C, Felicioni L, et al: EGFR mutations in non-small cell lung cancer: analysis of a large series of cases and development of a rapid and sensitive method for diagnostic screening with potential implications on pharmacologic treatment. J Clin Oncol 23: 857-865, 2005.

15. Minarik M, Gassman M, Belsanova B, et al: A novel highresolution chipCE assay for rapid detection of EGFR gene mutations and amplifications in lung cancer therapy by a combination of fragment analysis, denaturing CE and MLPA. Electrophoresis 31: 3518-3524, 2010.

16. Nagai Y, Miyazawa H, Huqun, et al: Genetic heterogeneity of the epidermal growth factor receptor in non-small cell lung cancer cell lines revealed by a rapid and sensitive detection system, the peptide nucleic acid locked nucleic acid PCR clamp. Cancer Res 65: 7276-7282, 2005.

17. Nomoto K, Tsuta K, Takano T, et al: Detection of EGFR mutations in archived cytologic specimens of non-small cell lung cancer using high-resolution melting analysis. Am J Clin Pathol 126: 608-615, 2006.

18. Oshita F, Matsukuma S, Yoshihara M, et al: Novel heteroduplex method using small cytology specimens with a remarkably high success rate for analysing EGFR gene mutations with a significant correlation to gefitinib efficacy in non-small cell lung cancer. Br J Cancer 95: 1070-1075, 2006.
19. Pan Q, Pao W and Ladanyi M: Rapid polymerase chain reactionbased detection of epidermal growth factor receptor gene mutations in lung adenocarcinomas. J Mol Diagn 7: 396-403, 2005.

20. Sasaki H, Endo K, Konishi A, et al: EGFR mutation status in Japanese lung cancer patients: genotyping analysis using LightCycler. Clin Cancer Res 11: 2924-2929, 2005.

21. Thomas RK, Nickerson E, Simons JF, et al: Sensitive mutation detection in heterogeneous cancer specimens by massively parallel picoliter reactor sequencing. Nat Med 12: 852-855, 2006.

22. Willmore-Payne C, Holden JA and Layfield LJ: Detection of epidermal growth factor receptor and human epidermal growth factor receptor 2 activating mutations in lung adenocarcinoma by high-resolution melting amplicon analysis: correlation with gene copy number, protein expression, and hormone receptor expression. Hum Pathol 37: 755-763, 2006.

23. Yatabe Y, Hida T, Horio Y, Kosaka T, Takahashi $T$ and Mitsudomi T: A rapid, sensitive assay to detect EGFR mutation in small biopsy specimens from lung cancer. J Mol Diagn 8: 335-341, 2006

24. Yu J, Kane S, Wu J, et al: Mutation-specific antibodies for the detection of EGFR mutations in non-small cell lung cancer. Clin Cancer Res 15: 3023-3028, 2009.

25. Zhou C, Zhao Y and Su B: Rapid detection of epidermal growth factor receptor mutations in non-small cell lung cancer using real-time polymerase chain reaction with TaqMan-MGB probes. Cancer J 12: 33-39, 2006.

26. Mitani Y, Lezhava A, Kawai Y, et al: Rapid SNP diagnostics using asymmetric isothermal amplification and a new mismatchsuppression technology. Nat Methods 4: 257-262, 2007.

27. Araki T, Shimizu K, Nakamura K, et al: Usefulness of peptide nucleic acid (PNA)-clamp smart amplification process version 2 (SmartAmp2) for clinical diagnosis of KRAS codon12 mutations in lung adenocarcinoma. Comparison of PNA-clamp SmartAmp2 and polymerase chain reaction-related methods. J Mol Diagn 12: $118-124,2009$.

28. Hoshi K, Takakura H, Mitani Y, et al: Rapid detection of epidermal growth factor receptor mutations in lung cancer by the Smart-amplification process. Clin Cancer Res 13: 4974-4983, 2007.

29. Cao W, Hashibe M, Rao JY, Morgenstern H and Zhang ZF: Comparison of methods for DNA extraction from paraffinembedded tissues and buccal cells. Cancer Detect Prev 27: 397-404, 2003.

30. Miyamae Y, Shimizu K, Mitani Y, et al: Mutation detection of epidermal growth factor receptor and KRAS genes using the smart amplification process version 2 from formalin-fixed, paraffin-embedded lung cancer tissue. J Mol Diagn 12: 257-264, 2010.

31. Ji H, Li D, Chen L, et al: The impact of human EGFR kinase domain mutations on lung tumorigenesis and in vivo sensitivity to EGFR-targeted therapies. Cancer Cell 9: 485-495, 2006.

32. Maheswaran S, Sequist LV, Nagrath S, et al: Detection of mutations in EGFR in circulating lung-cancer cells. N Engl J Med 359: 366-377, 2008.

33. Otani H, Toyooka S, Soh J, et al: Detection of EGFR gene mutations using the wash fluid of CT-guided biopsy needle in NSCLC patients. J Thorac Oncol 3: 472-476, 2008.

34. Soh J, Toyooka S, Aoe K, et al: Usefulness of EGFR mutation screening in pleural fluorescent substance fluid to predict the clinical outcome of gefitinib-treated patients with lung cancer. Int J Cancer 119: 2353-2358, 2006. 